\title{
A PROMETHEE II-BELIEF APPROACH FOR MULTI-CRITERIA DECISION-MAKING PROBLEMS WITH INCOMPLETE INFORMATION
}

\author{
DOI: $10.22367 / \operatorname{mcdm} .2021 .16 .04$
}

Received: 29.06.2021 | Revised: 3.09.2021 | Accepted: 29.11.2021.

\begin{abstract}
Multi-criteria decision aid methods consider decision problems in which many alternatives are evaluated on several criteria. These methods are used to deal with perfect information. However, in practice, it is obvious that this information requirement is too strict. In fact, the imperfect data provided by more or less reliable decision makers usually affect decision results, since any decision is closely linked to the quality and availability of information. In this paper, a PROMETHEE II-BELIEF approach is proposed to help multi-criteria decisions based on incomplete information. This approach solves problems with incomplete decision matrix and unknown weights within PROMETHEE II method. On the basis of belief function theory, our approach first determines the distributions of belief masses based on PROMETHEE II's net flows, and then calculates weights. Subsequently, it aggregates the distribution masses associated with each criterion using Murphy's modified combination rule in order to infer a global belief structure. The final alternative ranking is obtained via pignistic probability transformation. A case study of a real-world application concerning the location of a treatment center of waste from healthcare activities with infectious risk in the center of Tunisia is studied to illustrate the detailed process of the PROMETHEE II-BELIEF approach.
\end{abstract}

Keywords: multiple criteria aid, incomplete information, PROMETHEE II method, belief function theory.

OLID Research Laboratory, University of Sfax, Higher Institute of Industrial Management of Sfax, Road of Tunis km 10.5, Technopole of Sfax - BP 1164, Sfax 3021, Tunisia, e-mail: hela_frikha_moalla@escs.usf.tn, ORCID: 0000-0002-0233-697X.

** OLID Research Laboratory, University of Sfax, Higher Institute of Industrial Management of Sfax, Road of Tunis km 10.5, Technopole of Sfax - BP 1164, Sfax 3021, Tunisia, e-mail: ahmed.frikha@isgis.usf.tn, ORCID: 0000-0001-9772-853X. 


\section{Introduction}

Multi-criteria decision making deals with choosing, ranking or sorting alternatives on the basis of qualitative or quantitative criteria and preference judgments expressed by the decision maker. The literature presents many methods in decision analysis which come up with a satisfying decision. However, in order to be implemented, these methods assume that perfect information is available. That means the evaluations of the alternatives on the criteria as well as the preference parameters are known as exact numbers. Nevertheless, in practice, missing evaluations or imprecise information can occur. The information provided by the decision maker is usually imperfect because of its subjectivity. In fact, subjective information provided directly by the decision maker can hardly be applied successfully. The information imperfection includes the aspects of inconsistency, imprecision, incompleteness and uncertainty.

In this study, we will focus on the incompleteness of information. The incomplete information results from limited precision of human assessments which reduces its effectiveness in many applications. It may alter the final decision in practical situations, thus resulting in a gap between theory and practice. This gap is due to the fact that decision maker's preferences are not yet structured enough in his mind to allow the application of the decision making methods. For example, the decision maker cannot supply exact estimations of some parameters or he is not willing or able to define a stable preference structure or his complete evaluations of the consequences in the way required by the method. This inability might be due to his indisposition or his fear to decide exactly (Weber, 1987). Kim and Ahn (1999) claim that the possible reasons of the incompleteness of information provided by the decision maker are: (1) a decision should be made under time pressure and lack of data, (2) many criteria are intangible, as they reflect social and environmental impacts, (3) the decision maker has limited attention and information processing capability (Kahneman, Slovic and Tversky, 1982; Park et al., 1996), and (4) all group members do not have the same expertise in the given field (Ramanathan and Ganesh, 1994).

In this paper, we consider the PROMETHEE II method in which some evaluations of alternatives with respect to each criterion in the decision matrix are missing and criteria weights are unknown. In order to model the information incompleteness, the approach developed in this paper incorporates belief function theory with PROMETHEE II, the well known multi-criteria aggregation method. PROMETHEE II (Brans and Vincke, 1985; Brans, Vincke and Mareschal, 1986) is designed to solve complex problems involving multiple criteria. It presents several advantages and is considered a simple and clear 
method. It can also manage quantitative and qualitative criteria simultaneously and can solve the problem of incommensurability of measurement units (Sen et al., 2015). However, the standard PROMETHEE has many drawbacks. One of them is that it is time-consuming due to the high number of comparisons to be performed before any ranking can be evaluated; the number of comparisons rises quickly with the number of alternatives and criteria (Tscheikner-Gratl et al., 2017). Moreover, there is no allowance for ignorance with respect to types of alternatives and available criteria. It is also difficult for the decision maker to fix the shape of the criteria function and of parameter values (criteria weights, preference and indifference thresholds) (Sen et al., 2015). Indeed, the information concerning the parameter values provided by the decision maker is subjective and not very reliable, since it is based only on his experience, his intuition and his psychological state (Moalla Frikha, Chabchoub and Martel, 2011). Furthermore, the standard PROMETHEE II deals with perfect information only and not with incomplete, uncertain or imprecise one.

In order to alleviate these difficulties, we propose in this paper an approach integrating belief function theory into the PROMETHEE II method. Belief function theory (Dempster, 1967; Shafer, 1976) is a useful tool for representing and managing imperfect knowledge; it provides a suitable framework for dealing with incomplete data. In addition, it presents the advantage of combining distribution masses and taking decisions.

Incompleteness of information in the PROMETHEE II method can appear at different levels. Indeed, PROMETHEE II relies not only on the evaluations of alternatives with respect to criteria, but also on preference parameters, such as preference functions' thresholds and criteria weights. In our approach we focus on the incomplete decision matrix and unknown weights. The incompleteness of the decision matrix derives either from the decision maker's ignorance of some evaluations from the beginning or from the combination of alternatives having similar evaluations for a given criterion.

The paper is organized as follows: A literature review related to this research is presented in Section 2. Section 3 is devoted to a description of the PROMETHEE II method. A brief presentation of belief function theory is defined in Section 4. Section 5 develops the proposed PROMETHEE-BELIEF approach. In section 6, some examples are introduced to illustrate our approach and compare it with standard PROMETHEE. A case study of a real-world application related to management of waste from healthcare activities with infectious risk is included in Section 7. Section 8 concludes the paper and outline further research. 


\section{A literature review}

In the literature, several studies were carried out to help decisions based on imperfect information. For instance, Tacnet proposed ER-MCDA (Tacnet, 2009; Tacnet, Batton-Hubert and Dezert, 2009; Tacnet, Batton-Hubert and Dezert, 2010) to handle imprecise and uncertain information through a combination of Analytic Hierarchy Process (AHP) and belief function theory. In addition, Dezert et al. (2010) introduced imprecise evaluations of subsets and new discounting techniques. Thereafter, Tacnet and Dezert (2011) developed the COWA-ER for decision making under uncertainty to take into account imperfect evaluations of alternatives and unknown beliefs about groups of possible scenarios. Moreover, Hyde, Mayer and Colby (2003) proposed generalized criterion functions incorporated in PROMETHEE in order to take the uncertainty in the criteria performance values into consideration. Likewise, Pelissari et al. (2019) proposed a new method for sorting decision-making problems capable of dealing with multiple imperfect data and with criteria weight elicitation. Also, Ennaceur, Eloudei and Lefèvre (2012) extended the AHP method to an uncertain environment, where the uncertainty is represented through the Transferable Belief Model (TBM) in both the criterion and the alternative levels. Furthermore, Abdennadher, Boujelben and Ben Amor (2013) were interested in the PROMETHEE method where the alternatives are evaluated on a set of ordinal criteria and where the evaluations can be uncertain and imprecise. In addition, Ennaceur, Eloudei and Lefèvre (2014; 2016) and Altieri et al. (2017) proposed an extension of the belief Analytic Hierarchy Process (AHP) method based on the belief function where information is uncertain and imprecise. Later, Chen et al. (2017) suggested a novel method, based on the Dempster-Shafer evidence theory and Analytic Hierarchy Process, to handle the dependence in human reliability analysis. Their model can deal with uncertainty in an analyst's judgment and reduce the subjectivity in the evaluation process. Furthermore, Dezert, Han and Tacnet (2017) integrated belief functions into TOPSIS and proposed Imp-BF-TOPSIS to deal with imprecise score values (intervals of real numbers). Besides uncertainty and imprecision, various papers discussed incompleteness, the third aspect of imperfection. Several methods solve this type of problems using two steps procedures. In the first step, they proceed by completing the missing values in the decision matrix through applying a learning process (Morad, Svrcek and McKay, 2000; Hong, Tseng and Wang, 2002; Fortes et al., 2006) or heuristic rules (Raymond, 1986; Kaufman, 1988; Quinten and Raaijmakers, 1999; Quinlan, 1993), or by removing the alternatives or criteria with incomplete information from the problem. In the last case, the problem structure becomes 
distorted. The second step consists in applying the multi-criteria method to solve the problem. All these methods present disadvantages. Heuristic methods lack scientific foundations, since they calculate the missing value through replacing it by the mean of all known values or by the most frequent value under the criterion. Learning methods present also the drawback of complexity in their application to incomplete decision matrix.

Many other methods dealing with multi-criteria problems with incomplete information were developed on scientific basis. Weber (1987) presented an overview of existing methods which are particularly suitable for handling incomplete information. Thereafter, several approaches have been developed to make multi-criteria group decision under incomplete data (Kim and Ahn, 1997; 1999; Kim, Choi and Kim, 1999; Ju, 2014). Also, Hua, Gong and Xu (2008) proposed the DS-AHP approach for the multi-criteria decision making problems with incomplete decision matrix. This approach first identifies all possible focal elements with the incomplete decision matrix, and then calculates the basic probability assignment of each focal element. Next, the belief interval of each decision alternative is evaluated using belief function theory. Subsequently, preference relations are determined by comparing belief intervals. Moreover, Ren and Lutzen (2017) developed a novel multi-criteria decision-making method that combines Dempster-Shafer theory and a trapezoidal fuzzy Analytic Hierarchy Process for alternative energy source selection under incomplete information conditions. Likewise, Haseli, Sheikh and Shib (2020) proposed the Base-Criterion Method, which is capable to find lost comparisons in the worst terms of the incomplete pairwise comparison matrix between base-criterion and other criteria. Moreover, Fan and Deer (2005) developed a new approach to determine the parameter $\rho$ using belief function theory under incomplete information. In order to calculate the expected utility, an evaluation about the value of the parameter $\rho$ must be known. The authors assume that in the case of absence of evidence available about this value, the decision maker must provide partial information about it. This incomplete information is introduced into the developed model to solve the decision-making problem. Furthermore, Ben Amor and Mareschal (2012) proposed an approach to solve decision problems under incomplete decision matrix, using different models of the imperfection representation, that is, probabilities, fuzzy logic and possibility theory. In addition, Ahn (2015) presented a method dealing with incomplete attribute weights using extreme points.

For multi-criteria decision problems with incomplete information, the majority of papers dealt with the AHP method and integrated belief function theory (Beynon, Curry and Morgan, 2000; Hua, Gong and Xu, 2008; Wang, 
2006; Hsu and Wang, 2011; Huang et al., 2014; Ju, 2014). However, in our paper we are interested in incorporating belief function theory into the PROMETHEE method.

\section{The PROMETHEE approach}

PROMETHEE (Preference Ranking Organization Method for Enrichment Evaluations) (Brans and Vincke, 1985) is a multi-criteria decision making method for ranking alternatives evaluated on several conflicting criteria according to a decision maker's preferences. It is characterized by its simplicity and clearness (Brans, Vincke and Mareschal, 1986). Therefore, it has been applied in various area including environment, management, hydrology, business, finance, logistics and transportation, energy, manufacturing, and other fields (Behzadian et al., 2010). PROMETHEE is based on the principle of pairwise comparison of alternatives with respect to each criterion. The PROMETHEE methods involve five steps:

Step 1: Calculation of the performance differences from the decision matrix: The performance difference between each pair of alternatives $a_{i}$ and $a_{j}$ with respect to each criterion $k$ is calculated as follows:

$$
d_{i j}^{k}=\left\{\begin{array}{l}
g_{k}\left(a_{i}\right)-g_{k}\left(a_{j}\right) \text { for a criterion to maximize } \\
g_{k}\left(a_{j}\right)-g_{k}\left(a_{i}\right) \text { for a criterion to minimize }
\end{array}\right.
$$

where $g_{k}\left(a_{i}\right)$ and $g_{k}\left(a_{j}\right)$ show the performance of the alternatives $a_{i}$ and $a_{j}$, respectively, with regard to criterion $k$.

Step 2: A preference function $P_{i j}^{k}$ has to be associated with each criterion to model the decision-maker's preferences with respect to each criterion $k$. When the decision maker compares two alternatives $a_{i}$ and $a_{j}, P_{i j}^{k}$ represents the intensity of preference for $a_{i}$ over $a_{j}$, considering only the criterion $k$. The preference function's value varies between 0 and 1 and is assessed differently according to the criterion shape. The authors of PROMETHEE proposed six shapes of criteria functions that seem to cover most of the needs occurring in the real world (usual criterion, quasi criterion, criterion with linear preference, level criterion, criterion with linear preference and indifference area and Gaussian criterion). Depending on the manner in which the DM's preference increases with the difference between assessments of alternatives $a_{i}$ and $a_{j}$ with respect to the criterion $k$, the DM fixes the form of $P_{i j}^{k}$ and the associated parameters for every criterion. 
To define the preference function, it is necessary to fix the values of indifference thresholds $\left(q_{k}\right)$, preference thresholds $\left(p_{k}\right)$ and inflexion point of the Gaussian curve (Gaussian threshold's $\sigma_{k}$ ).

Step 3: Calculation of the aggregated preference index $\pi_{i j}$ : For each pair of alternatives, an aggregated preference index is calculated as follows:

$$
\pi_{i j}=\sum_{k=1}^{n} w_{k} P_{i j}^{k}
$$

where $w_{k}$ is the relative importance coefficient given to each criterion $k$ with $w_{k} \geq 0$ and $\sum_{k=1}^{n} w_{k}=1$. The greater $w_{k}$, the more important the criterion.

The aggregated preference index represents the degree of preference for $a_{i}$ over $a_{j}$ with respect to all the criteria simultaneously.

Step 4: Calculation of outranking flows: For each alternative, when compared with $(m-1)$ other alternatives, a positive, a negative and a net flow are calculated as:

$$
\begin{gathered}
\phi_{i}^{+}=\frac{1}{m-1} \sum_{j \neq i} \pi_{i j} \\
\phi_{i}^{-}=\frac{1}{m-1} \sum_{j \neq i} \pi_{j i}
\end{gathered}
$$

where $\phi_{i}^{+}$and $\phi_{i}^{-}$denote the positive and negative flows, respectively, for alternative $a_{i}$. A positive flow indicates the strength of $a_{i}$ with regard to other alternatives. Similarly, a negative flow indicates the weakness of $a_{i}$ with regard to other alternatives.

The net outranking flow $\phi_{i}$ is the difference between the outgoing and the incoming flows. It is obtained as follows:

$$
\phi_{i}=\phi_{i}^{+}-\phi_{i}^{-}
$$

Step 5: Alternative ranking

The solution of a particular decision problem depends on accepting or not the incomparability. If we accept it, we choose PROMETHEE I; otherwise, PROMETHEE II. PROMETHEE I generally leads to a ranking of the 
alternatives by a partial pre-order, since it accepts the incomparability, whereas PROMETHEE II leads to a ranking of alternatives by a total pre-order, as it does not accept the incomparability. According to PROMETHEE II, all the alternatives are ranked from the best to the worst one. In fact, $\phi_{i}$ can be positive or negative. The larger $\phi_{i}$, the more $x_{i}$ outranks the other alternatives, and the less it is outranked. Thus:

- $a_{i}$ outranks $a_{j}$ if and only if $\phi_{i}>\phi_{j}$ and

- $a_{i}$ is indifferent to $a_{j}$ if and only if $\phi_{i}=\phi_{j}$

Instead of calculating the aggregated preference index and multi-criteria flows (steps 3 and 4), we can simply calculate uni-criteria flows, that means the positive, negative and net flows for each alternative with respect to each criterion separately. These flows are as follows:

$$
\begin{gathered}
\phi_{i}^{k+}=\frac{1}{m-1} \sum_{j \neq i} P_{i j}^{k} \quad \forall k=1, \ldots, n \\
\phi_{i}^{k-}=\frac{1}{m-1} \sum_{j \neq i} P_{j i}^{k} \quad \forall k=1, \ldots, n \\
\phi_{i}^{k}=\phi_{i}^{k+}-\phi_{i}^{k-}
\end{gathered}
$$

The multi-criteria net flow is obtained as the weighted sum of mono-criteria net flows:

$$
\phi_{i}=\sum_{k=1}^{n} \phi_{i}^{k} w_{k}
$$

Alternative are ranked in decreasing order of their net flow values (step 5).

\section{Belief function theory}

The belief function theory (Dempster, 1967; Shafer, 1976) is a general framework for modeling uncertainty and imprecision when the available information is imperfect. It is an interesting tool for information fusion and decision making using, combination and decision rules, respectively.

A belief function model is defined by a finite and exhaustive set $\Theta$ called the frame of discernment of the problem under consideration. The set of all subsets of $\Theta$ is called the power set of $\Theta$ and denoted by $2^{\Theta}$.

A Basic Probability Assignment function (BPA) is a mapping $m: 2^{\Theta} \rightarrow[0,1]$. It assigns to every subset $A \subseteq \Theta$ a number $m(A)$, called the mass of $A$, which represents the degree of belief attributed exactly to $A$, and to none of its subsets. This function must satisfy the following conditions: $m(\varnothing)=0$, and $\Sigma\{m(A) / A \subseteq \Theta\}=1$. 
When $m(A)>0, A$ is called a focal element of $m$. The set of focal elements of $m$ is denoted $\mathfrak{I}$ and the pair $(\mathfrak{I}, m)$ is called the body of evidence.

A BPA can be represented equivalently by its associated belief and plausibility functions. A belief function is a mapping Bel: $2^{\Theta} \rightarrow[0,1]$, defined as:

$$
\operatorname{Bel}(A)=\sum_{B \subseteq A} m(B) \quad \forall A \subseteq \Theta
$$

$\operatorname{Bel}(A)$ measures the total belief (credibility) completely committed to $A \subseteq \Theta$. A plausibility function is a mapping $P l: 2^{\Theta} \rightarrow[0,1]$, defined as:

$$
P l(A)=\sum_{B \cap A \neq \varnothing} m(B) \quad \forall A \subseteq \Theta
$$

$P l(A)$ can be regarded as the maximum amount of belief that could be given to $A$.

In belief function theory, combination is an operation that plays a fundamental role. The BPAs generated by several distinct sources are combined to yield a global BPA that synthesizes the data provided by the different sources.

Let $m_{i}$ and $m_{j}$ denote two BPAs obtained from two distinct sources $i$ and $j$ in the same frame of discernment $\Theta$. According to Dempster's rule of combination (Shafer, 1976), we have:

$$
m(A)=\frac{1}{1-K} \sum_{B \cap C=A} m_{i}(B) \times m_{j}(C) \forall A \subseteq \Theta, \text { where } K=\sum_{B \cap C=\varnothing} m_{i}(B) \times m_{j}(C)
$$

Dempster's combination rule verifies some interesting properties (commutative and associative). However, in some situations, this rule cannot be used. When there are large conflicts between bodies of evidence, counterintuitive behavior will emerge (Zadeh, 1979). Other rules have been proposed to deal with the inconvenience of the loss of majority opinion. For instance, Murphy (2000) has, proposed, for the first time, the average rule where the belief mass of a subset $A \subseteq \Theta$ provided by independent sources are averaged to determine the global belief mass on $A$. Suppose there are $n$ information sources providing $n$ BPAs $m_{i}$ for all $i=1, \ldots, n$. The average rule is defined as:

$$
m(A)=\frac{1}{n} \sum_{i=1}^{n} m_{i}(A) \forall A \in \Theta \text { and } A \neq \varnothing
$$

This rule does not, however, offer convergence toward certainty. In fact, it is not always adequate to yield reasonable results, particularly when the evidence has a high degree of conflict. For this reason, Murphy has proposed another combination rule based on the idea of incorporating the average operation into Dempster's rule of combination. In this rule, all information sources are equally important, and, therefore, have the same weight $(1 / n)$ in the sum of evidence (equation 13). Murphy's averaging rule is recommended in cases when the 
objective is to preserve the opinion of the majority when one source contradicts several other, consistent sources.

In belief function theory, several decision rules are possible; they are, most of the times, applied to the choice of one hypothesis from among many. In order to obtain a decision without ambiguity, we should choose the hypothesis whose credibility is superior to the plausibility of any other hypothesis. Nonetheless, credibility and plausibility functions may generate, in several situations, different ranking of a single hypothesis. To overcome this inconvenience, other decision rules have been developed, based on either credibility or plausibility, such as the maximum of credibility and the maximum of plausibility. The first rule selects the hypothesis with the maximum of total belief and the second chooses the least contradicted hypothesis. However, the maximum of credibility has the drawback of not being used when focal elements are sets of hypothesis. Furthermore, Smets (2002) claims that the maximum of plausibility decision rule is subject to counterexamples. For that purpose, he transforms belief functions into a pignistic probability function BetP to make decisions. This transformation consists of distributing each mass $m(A)$ equally among the statements that compose $A \subseteq \Theta$. Formally, BetP is defined as:

$$
\operatorname{BetP}(A)=\sum_{B \subseteq A} m(B) \frac{|A \cap B|}{|B|} \quad \forall A \subseteq \Theta
$$

where $|B|$ is the cardinality of $B . \operatorname{Bet} P(A)$ can be viewed as a betting commitment to $A$ and represents the total mass value that $A$ can carry. The decision rule used consists in choosing the hypothesis with the maximum of pignistic probability.

$$
\theta_{\text {likely }}=\operatorname{Arg} \underset{\theta_{i} \in \Theta}{\operatorname{Max}}\left(\operatorname{BetP}\left(\theta_{i}\right)\right)
$$

\section{The PROMETHEE II-BELIEF approach}

Let $P=\{A, C, g, f\}$ be a multi-criteria decision problem where $A=\left\{a_{1}, a_{2}, \ldots, a_{m}\right\}$ is a non-empty finite set of alternatives $a_{i}$ and $C=\left\{c_{1}, c_{2}, \ldots, c_{n}\right\}$ is a non-empty finite set of decision criteria $c_{k}$. For each criterion $c_{k}, f: A \times C \rightarrow G$, $f\left(a_{i}, c_{k}\right) \in G$, where $g_{k}\left(a_{i}\right)$, an element of $G$, is called the evaluation of alternative $i$ with respect to criterion $k$. If there is at least one criterion $c_{k}$ and $g_{k}\left(a_{i}\right)$ which includes missing values, then problem $P$ is called a multi-criteria decision making problem with incomplete information. Missing values in the decision matrix are denoted by asterisks, as are the unknown weights $w_{k}$ associated with criterion $k$. For each criterion, the decision maker must provide at least two distinct evaluations of alternatives. 
In the proposed PROMETHEE II-BELIEF, we first determine the focal elements, and then the distributions of belief masses using PROMETHEE's net flows. Thereafter, weights are calculated and the obtained distribution masses are combined using Murphy's modified combination rule in order to infer a global belief structure. The ranking of alternatives is based on pignistic probabilities.

\subsection{Determination of focal elements from the incomplete decision matrix}

Let $A=\left\{a_{1}, a_{2}, \ldots, a_{m}\right\}$ be the set of decision alternatives, equivalent to the frame of discernment, and $A_{h}\left(h=1, \ldots, 2^{m}\right)$ be a subset of $A$. Each subset $A_{h} \subseteq A$ such that $g_{k}\left(A_{h}\right)>0$ is called a focal element, which can be defined from the decision matrix as follows:

Definition 1. For all $a_{i}$ and $a_{j} \in A$ with $a_{i} \neq a_{j}$, if $g_{k}\left(a_{i}\right)=g_{k}\left(a_{j}\right)$, then $a_{i}$ and $a_{j}$ belong to the same focal element. Hence, both alternatives $a_{i}$ and $a_{j}$ are regrouped under one focal element.

Hence, from the decision matrix $G$ we generate a new decision matrix $G^{\prime}=g_{k}^{\prime}\left(A_{h}\right)$, where $g_{k}^{\prime}\left(A_{h}\right)$ is the evaluation of the subsets of alternatives $A_{h}$ with respect to each criterion $c_{k}\left(k=1, \ldots, n ; h=1, \ldots, p ; p \leq 2^{m}\right)$. The matrix $G$ ' does not contain all the $2^{m}$ subsets, but only the focal elements $A_{h}$. This step can considerably reduce the number of comparisons within the PROMETHEE II method.

\subsection{Determination of belief mass distributions}

Using the decision matrix $G^{\prime}$, we apply the PROMETHEE II method, which consists in comparing not only decision alternatives, but also subsets of alternatives (focal elements) $A_{h}$ and $A_{l}$ with $h \neq l$, pairwise with respect to each criterion $k$. We calculate the preference function values and then the uni-criteria positive, negative and net flows of each subset $A_{h}$ for each criterion separately (equations 6-8).

In the PROMETHEE II method, net flows can be positive or negative. In order to transform net flows into belief mass distributions, and since the belief mass $m_{k}\left(A_{h}^{k}\right)$ must be positive, we first calculate an exponential function $\lambda^{\phi_{h}^{k}}$ for each net flow $\phi_{h}^{k}$ for $h=1, \ldots, p ; p \leq 2^{m} ; k=1, \ldots, n$. The base of the exponential function is $\lambda$.

If $\lambda$ is positive and smaller than $1, \lambda^{\phi_{h}^{k}}$ is a decreasing function. However, the belief mass distribution based on $\lambda^{\phi_{h}^{k}}$ must be an increasing function of the net flow. Hence, the base should be greater than 1 . The bigger the base $\lambda$, the faster the exponential function shrinks for low values of net flow. 
Next, the values of $\lambda^{\phi_{h}^{k}}$ must be normalized in order to obtain belief mass distributions for all the focal elements with respect to each criterion.

Definition 2. For all focal elements $A_{h}^{k} \in 2^{A}$, the belief mass distribution associated with each criterion $k$ is defined as:

$$
m_{k}\left(A_{h}^{k}\right)=\frac{\lambda^{\phi_{h}^{k}}}{\sum_{h=1}^{p} \lambda^{\phi_{h}^{k}}} \forall k=1, \ldots, n \text { and } \forall h=1, \ldots, p ; p \leq 2^{m}
$$

The normalization rule (equation 16) guarantees that $m_{k}\left(A_{h}^{k}\right) \in[0,1]$ and $\sum\left(m_{k}\left(A_{h}^{k}\right) / A_{h}^{k} \subseteq \mathcal{A}\right)=1$.

Since the belief mass distribution $m_{k}\left(A_{h}^{k}\right)$ is an increasing function of the net flow, the higher the net flow, the greater the mass and the more preferred the given subset. The belief mass measures the strength of the subset $A_{h}^{k}$ compared to all other subsets with respect to each criterion $k$. The more important the belief mass, the more focal element $A_{h}^{k}$ dominates the others.

\subsection{Criteria weight determination}

To solve the problem of data subjectivity while providing precise weight values, we propose to determine criteria weights based on belief mass distributions deduced from mono-criteria net flows. The new method relies on the difference between two pignistic probabilities associated with two belief masses.

Formally, let us consider $\operatorname{Bet}_{k}$ and $\operatorname{Bet}_{k}$, pignistic probabilities derived from belief masses associated respectively with criteria $k$ and $k^{\prime}$. The distance between betting commitments $\operatorname{Bet}_{k}$ and $\operatorname{Bet}_{k}$, (Liu, 2006) is denoted by $\operatorname{difBet} P$ and defined as:

$$
\operatorname{DifBetP}\left(k, k^{\prime}\right)=\underset{A_{h} \subseteq \mathfrak{J}}{\operatorname{Max}}\left(\left|\operatorname{Bet}_{k}\left(A_{h}\right)-\operatorname{Bet}_{k^{\prime}}\left(A_{h}\right)\right|\right)
$$

Definition 3. Assume $n$ pignistic probabilities generated from $n$ criteria. We define the similarity degree between two criteria $k$ and $k^{\prime}$ as:

$$
\operatorname{Sim}\left(k, k^{\prime}\right)=1-\operatorname{DifBeP}\left(k, k^{\prime}\right)
$$


Definition 4. The importance degree Imp of each criterion is defined as:

$$
\operatorname{Imp}(k)=\sum_{\substack{k=1 \\ k \neq k^{\prime}}}^{n} \operatorname{Sim}\left(k, k^{\prime}\right) \forall k=1, \ldots, n
$$

The more similar to other criteria criterion $k$ is, the more important it is.

Definition 5. The normalized weight vector $w_{k}$ of all criteria $k=1, \ldots, n$ is defined as:

$$
w_{k}=\frac{\operatorname{Imp}(k)}{\sum_{k=1}^{n} \operatorname{Imp}(k)}
$$

This transformation ensures that weight values are normalized so that $0 \leq w_{k} \leq 1$ and $\sum_{k=1}^{n} w_{k}=1$. Obviously, the higher the importance degree of a criterion, the higher its weight.

\subsection{Combination of belief mass distribution}

In the PROMETHEE II method, all mono-criteria net flows must be aggregated using weight values in order to get multi-criteria net flows. Similarly, in our proposed method, the aggregation operation involves combining belief mass distributions derived from mono-criteria net flows. The combination allows for extracting a global belief mass structure which is equivalent to a multi-criteria net flow.

To combine all deduced belief mass distributions, Murphy's rule is used. This rule considers all criteria as equally important, and therefore as having the same weight $(1 / n)$, which is not always reasonable in real-life cases since some criteria may be more important than others. Hence, criteria weights must be taken into consideration. We propose to modify Murphy's combination rule using the obtained objective weight values instead of equal weights. Thereafter, a modified Murphy's combination rule is applied to compute weighted average mass $\bar{m}$ as follows:

$$
\bar{m}\left(A_{h}\right)=\sum_{k=1}^{n}\left(w_{k} \times m_{k}\left(A_{h}\right)\right) \forall A_{h} \subseteq \mathcal{A}
$$

The weighted average mass must be combined $n-1$ times using Dempster's rule to obtain an overall belief mass $m$ :

$$
m\left(A_{h}\right)=\underbrace{\bar{m}\left(A_{h}\right) \oplus \cdots \oplus \bar{m}\left(A_{h}\right)}_{(n-1) \text { times }} \quad \forall A_{h} \subseteq \mathcal{A}
$$


Our proposed aggregation approach presents many advantages. Since in multicriteria problems criteria are conflicting and the determination of the best compromise solution is required, our proposed aggregation approach solves the problem of conflicting criteria by preserving the evaluation of the majority of criteria. In addition, it presents the advantage of considering criteria weights to calculate the overall belief mass distribution for each focal element. Finally, the global information obtained from modified Murphy's rule will be used for alternative ranking.

\subsection{Alternative ranking}

To rank alternatives from the best to the worst, we must first transform the obtained overall belief mass into pignistic probability BetP using pignistic transformation, defined as:

$$
\operatorname{BetP}\left(a_{i}\right)=\sum_{a_{i} \in A_{h}} \frac{m\left(A_{h}\right)}{\left|A_{h}\right|} \forall a_{i} \in \mathcal{A}
$$

This transformation allows to have a global evaluation for each alternative. The ranking of alternatives is performed in decreasing order of pignistic probabilities.

\section{Experimental settings}

In this section, a few examples are introduced to illustrate our approach and to compare it with the standard PROMETHEE II. We consider the case of a complete decision matrix with equal evaluations of some alternatives, the particular cases where an alternative is dominated, cases where an alternative is dominant, and, finally, the case of an incomplete decision matrix.

\subsection{Complete decision matrix with some equal alternative evaluations}

In order to compare our proposed approach PROMETHEE II-BELIEF with the standard PROMETHEE II, we consider a multi-criteria illustrative example where the elements of the decision matrix are known. The decision matrix contains alternatives with the same evaluations according with respect to some criteria. The decision matrix $G$ is shown in Table 1. 
Table 1: Decision matrix $G$

\begin{tabular}{|c|c|c|c|c|c|}
\hline & $C_{1}$ & $C_{2}$ & $C_{3}$ & $C_{4}$ & $C_{5}$ \\
\hline$A$ & 20 & 15 & 4 & $\underline{3}$ & $\underline{3}$ \\
\hline$B$ & 52 & 20 & 3 & 4 & $\underline{3}$ \\
\hline$C$ & 14 & 35 & 2 & 2 & 4 \\
\hline$D$ & 5 & 63 & $\underline{1}$ & 1 & 2 \\
\hline$E$ & 8 & 10 & $\underline{1}$ & $\underline{3}$ & 1 \\
\hline
\end{tabular}

The shapes of the criteria as well as their associated parameters (indifference thresholds $q_{k}$, preference thresholds $p_{k}$ and Gaussian threshold $\sigma_{k}$ ) are:

- $C_{1}$ is a Gaussian criterion with $\sigma_{1}=10$

- $C_{2}$ is a level criterion with $q_{2}=5$ and $p_{2}=10$

- $C_{3}$ is a criterion with linear preference and indifference area with $q_{3}=0.5$ and $p_{3}=1.5$

- $C_{4}$ is a level criterion with $q_{4}=1$ and $p_{4}=1.5$

- $C_{5}$ is a quasi criterion with $q_{5}=1$

\section{PROMETHEE II-BELIEF}

In order to apply PROMETHEE II-BELIEF, decision matrix $G$ ' evaluating focal elements, belief mass distributions as well as criteria weight values are presented in appendix A (Table A.1-A.3).

The obtained weight values are introduced to modified Murphy's rule to combine all belief mass distributions. Thereafter, we transform the obtained overall belief mass into pignistic probabilities BetP using pignistic transformation. The results are as follows:

$\operatorname{BetP}(A)=0.4527 ; \operatorname{Bet} P(B)=0.2752 ; \operatorname{Bet} P(C)=0.1115 ; \operatorname{Bet} P(D)=0.0286 ;$ $\operatorname{BetP}(E)=0.1320$

The alternatives are ranked in decreasing order of their pignistic probabilities. We obtain the following ranking: $A>B>E>C>D$.

\section{Standard PROMETHEE II}

We integrate the weight values obtained from PROMETHEE II-BELIEF into the standard PROMETHEE II. The following net flows are then obtained:

$$
\phi_{A}=0.4590 ; \phi_{B}=0.2141 ; \phi_{C}=-0.1556 ; \phi_{D}=-0.6460 ; \phi_{E}=0.1285
$$

According to the net flows values, we rank the alternatives from the best to the worst. We obtain the following ranking: $A>B>E>C>D$ 


\subsection{Particular case: Dominant alternative}

In this example (Table 2), we assume that alternative $B$ is dominant over all criteria.

Table 2: Decision matrix with a dominant alternative

\begin{tabular}{|c|c|c|c|c|c|}
\hline & $C_{1}$ & $C_{2}$ & $C_{3}$ & $C_{4}$ & $C_{5}$ \\
\hline$A$ & 20 & 15 & 3 & $\underline{3}$ & $\underline{5}$ \\
\hline $\boldsymbol{B}$ & $\mathbf{4}$ & $\mathbf{8}$ & $\mathbf{4}$ & $\mathbf{4}$ & $\underline{\mathbf{5}}$ \\
\hline$C$ & 14 & 35 & 2 & 2 & 4 \\
\hline$D$ & 5 & 63 & $\underline{1}$ & 1 & 2 \\
\hline$E$ & 8 & 10 & $\underline{1}$ & $\underline{3}$ & 1 \\
\hline
\end{tabular}

\section{PROMETHEE II-BELIEF}

Calculations based on PROMETHEE II's net flows, are presented in appendix B (Table B.1-B.3). The overall belief mass is transformed into pignistic probabilities $B e t P$, which are:

$\operatorname{BetP}(A)=0.2037 ; \operatorname{Bet} P(B)=0.6580 ; \operatorname{BetP}(C)=0.0582 ; \operatorname{Bet} P(D)=0.0121 ;$ $\operatorname{BetP}(E)=0.0680$

The alternatives are ranked according to the decreasing order of their pignistic probabilities. The obtained ranking is: $B>A>E>C>D$

\section{Standard PROMETHEE II}

Using the obtained weights, PROMETHEE II's net flows are calculated:

$$
\phi_{A}=0.2965 ; \phi_{B}=0.6874 ; \phi_{C}=-0.2257 ; \phi_{D}=-0.7826 ; \phi_{E}=0.0244
$$

We obtain the following ranking of the alternatives: $B>A>E>C>D$.

The results confirm the assumption of the particular case and show that alternative $B$ is dominant, either for the PROMETHEE II-BELIEF approach or the standard PROMETHEE II. In fact, both $\operatorname{Bet} P(B)$ and $\phi_{B}$ are far greater than other alternative values.

\subsection{Particular case: Dominated alternative}

In this example, we present a particular case where alternative $D$ is dominated by all other alternatives. It has high evaluations with respect to the criteria to be minimized and low evaluations with respect to the criteria to be maximized. The assessments are presented in Table 3. 
Table 3: Decision matrix with a dominated alternative

\begin{tabular}{|c|c|c|c|c|c|}
\hline & $C_{1}$ & $C_{2}$ & $C_{3}$ & $C_{4}$ & $C_{5}$ \\
\hline$A$ & 20 & 15 & 4 & $\underline{3}$ & $\underline{3}$ \\
\hline$B$ & 52 & 20 & 3 & 4 & $\underline{3}$ \\
\hline$C$ & 14 & 35 & 2 & 2 & 4 \\
\hline$D$ & $\mathbf{5 5}$ & $\mathbf{6 3}$ & $\underline{\mathbf{1}}$ & $\mathbf{1}$ & $\mathbf{1}$ \\
\hline$E$ & 8 & 10 & $\underline{1}$ & $\underline{3}$ & 2 \\
\hline
\end{tabular}

\section{PROMETHEE II-BELIEF}

Calculations using the PROMETHEE II-BELIEF approach are given in appendix C (Table C.1-C.3).

Pignistic probabilities BetP are:

$\operatorname{BetP}(A)=0.4667 ; \operatorname{Bet} P(B)=0.2443 ; \operatorname{Bet} P(C)=0.1202 ; \operatorname{Bet} P(D)=0.0062 ;$ $\operatorname{BetP}(E)=0.1626$

The ranking of the alternatives is: $A>B>E>C>D$

\section{Standard PROMETHEE II}

Applying the standard PROMETHEE II, we obtain the following net flows:

$$
\phi_{A}=0.5406 ; \phi_{B}=0.2539 ; \phi_{C}=-0.0869 ; \phi_{D}=-0.9939 ; \phi_{E}=0.2863
$$

The ranking of the alternatives is: $A>E>B>C>D$

We can clearly see that alternative $D$ is dominated by all other alternatives, either for our approach or for the standard PROMETHEE II. In fact, $\operatorname{Bet} P(D)$ has a negligible value close to 0 , while $\phi_{D}$ is very low.

In all cases, we obtain the same ranking of alternatives using either PROMETHEE II-BELIEF or the standard PROMETHEE II. Nevertheless, our proposed approach presents three advantages over the standard PROMETHEE II. First, it reduces considerably the number of pairwise comparisons by regrouping alternatives with the same evaluation under the same focal element. Second, PROMETHEE II-BELIEF allows to determine objective criteria weights on the basis of scientific foundations, hence reducing subjectivity. Third, this approach is capable of solving multi-criteria decision problems with an incomplete decision matrix, which is not feasible using the standard PROMETHEE II.

\subsection{Incomplete decision matrix}

In this example, we omit some alternative evaluations with respect to some criteria in order to obtain the following incomplete decision matrix (Table 4): 
Table 4: Incomplete decision matrix

\begin{tabular}{|c|c|c|c|c|c|}
\hline & $C_{1}$ & $C_{2}$ & $C_{3}$ & $C_{4}$ & $C_{5}$ \\
\hline$A$ & 20 & 15 & $*$ & 3 & 4 \\
\hline$B$ & 52 & $*$ & 3 & $*$ & 3 \\
\hline$C$ & 14 & 35 & 2 & 2 & $*$ \\
\hline$D$ & 5 & 63 & 4 & 1 & 2 \\
\hline$E$ & $*$ & 10 & 1 & 4 & 1 \\
\hline
\end{tabular}

\section{PROMETHEE II-BELIEF}

Appendix D illustrates the details of the calculations of belief masses and criteria weights for this example (Table D.1-D.3).

Pignistic probabilities BetP associated with each alternative are:

$\operatorname{Bet} P(A)=0.4012 ; \operatorname{Bet} P(B)=0.0206 ; \operatorname{Bet} P(C)=0.0735 ; \operatorname{Bet} P(D)=0.3447 ;$ $\operatorname{BetP}(E)=0.1600$

The ranking of the alternatives is: $A>D>E>C>B$

\section{Standard PROMETHEE II}

The standard PROMETHEE II cannot be applied when the decision matrix is incomplete.

\section{Real-world applications}

Since the management of waste from healthcare activities is of particular interest worldwide and, more specifically, in our Tunisian context, we focus in this paper on waste from healthcare activities with infectious risk. This interest originates from the fast development that recorded the structure of public and private health care in Tunisia and which has been accompanied by a corresponding increase in the number of patients treated, as well as the quantities of waste generated within health facilities. Because infectious waste is suspected to contain pathogens (bacteria, viruses, parasites or fungus) in sufficient concentration or quantity to cause disease in susceptible hosts, it has various irreversible impacts on public health and deleterious effects on the environment. Therefore, improvement in waste management was considered one of the most important concerns of the national system for the management of hazardous waste in Tunisia. An efficient management of hazardous waste consists in optimizing the location of undesirable facilities (the treatment, recycling and/or destruction plant). Location of undesirable plants is a complex process, because it combines social, environmental, political and technical objectives. 
In this paper, the potential of the PROMETHEE-BELIEF approach is illustrated by a real-life case study. The Ministry of Environment considers the problem of choosing the best site for installing a new waste treatment center for healthcare activities with infectious risk in the center of Tunisia. The potential sites are nine Tunisian cities: Sousse ( $a_{1}$ : industrial area of Kalaa Kebira), Monastir ( $a_{2}$ : industrial area of Jemmel), Mahdia ( $a_{3}$ : industrial area of Ksour essef), Sfax ( $a_{4}$ : industrial area of Hencha), Gabes $\left(a_{5}\right.$ : industrial area of south Gabes), Kairouan ( $a_{6}$ : Industrial area of Hajeb Layoun), Sidi Bouzid ( $a_{7}$ : Industrial area of Sidi Bouzid Ouest), Kasserine ( $a_{8}$ : Industrial area of North of Kasserine) and Gafsa ( $a_{9}$ : Industrial area of South of Gafsa) (see Figure 1). These decision alternatives are evaluated with respect to five criteria.

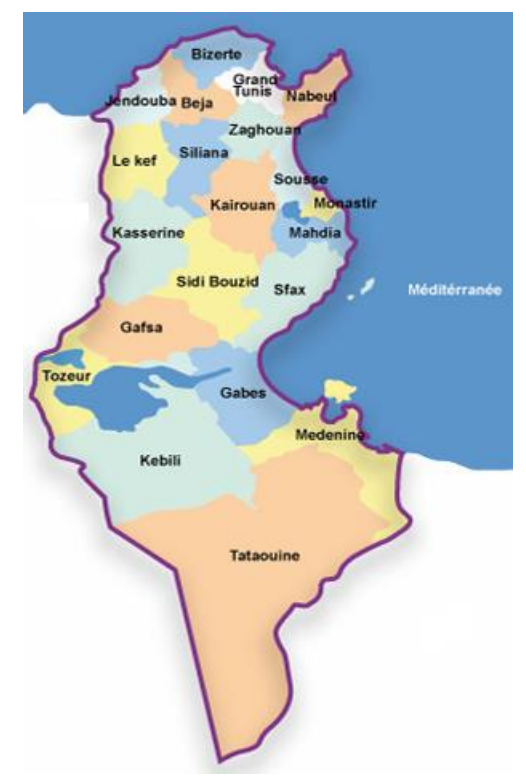

Figure 1: Map of Tunisia

- $C_{1}$ (Installation cost in millions of TND): It includes land acquisition costs, construction and civil engineering costs, labor and administrative costs and operating costs. The Ministry of Environment intends to choose the site that minimizes this cost. Therefore, installation cost is selected in this paper as a criterion to be minimized.

- $C_{2}$ (Population in the vicinity of the waste treatment center): Infectious waste should always be assumed to potentially contain a variety of pathogenic microorganisms. This is because the presence or absence of pathogens cannot be determined at the time a waste item is produced and discarded into a container. Pathogens in infectious waste that is not well managed may enter 
the human body through several routes. For this reason, the waste center presents risk to residents. Thus, it should be located in an area that is scarcely populated. Consequently, $C_{2}$ is a criterion to be minimized.

- $C_{3}$ (Quantity of waste of healthcare activities with infectious risk collected from all hospitals of the city, expressed in tons per year): The more waste healthcare facilities of the city generate, the greater the need to create a new center. Hence, quantity of waste is a criterion to be maximized.

- $C_{4}$ (Number of existing centers): The fewer treatment centers the city has, the greater the need to create new centers. Accordingly, $C_{4}$ is a criterion to be minimized.

- $C_{5}$ (Proximity to urban areas): Since treatment centers operate on waste from infectious healthcare activities, they present pollution drawbacks and risks to inhabitants and environment. Therefore, the new center should be located far from the city center, where the population is concentrated and soil and groundwater are intensively used. Alternative sites are evaluated with respect to this criterion on a 5-level scale. Level 1 and level 5 indicate that the potential waste treatment center is close and far from the city center, respectively. So, the fifth criterion is a criterion to be maximized.

The evaluations of alternative sites with respect to each criterion are presented in the following decision matrix (Table 5). In the industrial area of Hajeb Layoun in Kairouan city, the persons responsible for the project in the ministry of environment have not yet decided which location to choose because they have three proposals. Therefore, the installation cost in Kairouan remains unknown. Moreover, we fail to have the exact waste quantity since some hospitals, either in Monastir or in Gabes, present defaulting information systems. In addition, the population in the industrial area of Sidi Bouzid Ouest, as well as in the industrial area of North of Kasserine, is difficult to assess due to lack of information in municipalities of these cities. Subsequently, some evaluations are missing and the decision matrix is incomplete (asterisks denote the missing values).

Table 5: Incomplete decision matrix

\begin{tabular}{|l|c|c|c|c|c|}
\hline \multicolumn{1}{|c|}{ City } & Installation cost & Population & Waste quantity & Existing centers & $\begin{array}{c}\text { Proximity to } \\
\text { urban areas }\end{array}$ \\
\hline Sousse & 1350 & 51196 & 386 & 2 & 3 \\
Monastir & 1200 & 55272 & $*$ & 0 & 4 \\
Mahdia & 1200 & 48799 & 208 & 0 & 3 \\
Sfax & 1300 & 47170 & 460 & 3 & 5 \\
Gabes & 1350 & 61699 & $*$ & 0 & 1 \\
Kairouan & $*$ & 35403 & 113 & 1 & 5 \\
Sidi Bouzid & 850 & $*$ & 90 & 0 & 2 \\
Kasserine & 850 & $*$ & 104 & 0 & 2 \\
Gafsa & 900 & 90742 & 92 & 2 & 2 \\
\hline
\end{tabular}


In order to apply PROMETHEE II-BELIEF, we transform the decision matrix $G$ (evaluating alternatives) to another decision matrix $G^{\prime}$ (evaluating focal elements). We regroup the alternatives with the same evaluation with respect to a criterion under a focal element. The focal element decision matrix is presented in Table 6.

Table 6: Decision matrix of focal elements for the waste treatment center location problem

\begin{tabular}{|c|c|c|c|c|c|}
\hline City & $\begin{array}{c}\text { Installation } \\
\text { cost }\end{array}$ & Population & $\begin{array}{c}\text { Waste } \\
\text { quantity }\end{array}$ & $\begin{array}{c}\text { Existing } \\
\text { centers }\end{array}$ & $\begin{array}{c}\text { Proximity to } \\
\text { urban areas }\end{array}$ \\
\hline Sousse & $*$ & 51196 & 386 & $*$ & $*$ \\
\hline Monastir & $*$ & 55272 & $*$ & $*$ & 4 \\
\hline Mahdia & $*$ & 48799 & 208 & $*$ & $*$ \\
\hline Sfax & 1300 & 47170 & 460 & 3 & $*$ \\
\hline Gabes & $*$ & 61699 & $*$ & $*$ & $*$ \\
\hline Kairouan & $*$ & 35403 & 113 & 1 & $*$ \\
\hline Sidi Bouzid & $*$ & $*$ & 90 & $*$ & $*$ \\
\hline Kasserine & $*$ & $*$ & 104 & $*$ & $*$ \\
\hline Gafsa & 900 & 90742 & 92 & $*$ & $*$ \\
\hline Sousse $\cup$ Gabes & 1350 & $*$ & $*$ & $*$ & $*$ \\
\hline Monastir $\cup$ Mahdia & 1200 & $*$ & $*$ & $*$ & $*$ \\
\hline Sidi Bouzid $\cup$ Kasserine & 850 & $*$ & $*$ & $*$ & $*$ \\
\hline Sousse $\cup$ Gafsa & $*$ & $*$ & * & 2 & $*$ \\
\hline $\begin{array}{l}\text { Monastir } \cup \text { Mahdia } \cup \\
\text { Gabes } \cup \text { Sidi Bouzid } \cup \\
\text { Kasserine }\end{array}$ & $*$ & $*$ & $*$ & 0 & $*$ \\
\hline Sousse $\cup$ Mahdia & $*$ & $*$ & $*$ & $*$ & 3 \\
\hline Sfax $\cup$ Kairouan & $*$ & $*$ & $*$ & $*$ & 5 \\
\hline Gabes $\cup$ Kasserine & $*$ & $*$ & $*$ & $*$ & 1 \\
\hline Sidi Bouzid $\cup$ Gafsa & $*$ & $*$ & $*$ & $*$ & 2 \\
\hline
\end{tabular}

The criteria shapes and their associated parameters are defined as:

- Installation cost is a level criterion with $q_{1}=100$ and $p_{1}=300$.

- Population is a criterion with linear preference and indifference area with $q_{2}=$ $=10000$ and $p_{2}=20000$.

- Waste quantity is a level criterion with $q_{3}=20$ and $p_{3}=100$.

- Existing centers is a usual criterion.

- Proximity to urban areas is a quasi criterion with $q_{5}=3$.

We apply the PROMETHEE II method based on $G$, then we determine the uni-criteria net flows associated with each focal element with respect to each criterion, and then we apply equation 16 to determine the belief mass distribution for each focal element with respect to each criterion. We assign the value 2 to the parameter $\lambda$ because it must be greater than 1 and should not have a high value. These belief masses are given in Table 7. 
Table 7: Belief mass distributions for the waste treatment center location problem

\begin{tabular}{|c|c|c|c|c|c|}
\hline City & $\begin{array}{c}\text { Installation } \\
\text { cost }\end{array}$ & Population & $\begin{array}{c}\text { Waste } \\
\text { quantity }\end{array}$ & $\begin{array}{l}\text { Existing } \\
\text { centers }\end{array}$ & $\begin{array}{l}\text { Proximity to } \\
\text { urban areas }\end{array}$ \\
\hline Sousse & 0 & 0.0571 & 0.3171 & 0 & 0 \\
\hline Monastir & 0 & 0.0416 & 0 & 0 & 0.1818 \\
\hline Mahdia & 0 & 0.0796 & 0.0396 & 0 & 0 \\
\hline Sfax & 0.1710 & 0.0997 & 0.6341 & 0.0118 & 0 \\
\hline Gabes & 0 & 0.0238 & 0 & 0 & 0 \\
\hline Kairouan & 0 & 0.6976 & 0.0050 & 0.1882 & 0 \\
\hline Sidi Bouzid & 0 & 0 & 0.0012 & 0 & 0 \\
\hline Kasserine & 0 & 0 & 0.0018 & 0 & 0 \\
\hline Gafsa & 0.3879 & 0.0006 & 0.0012 & 0 & 0 \\
\hline Sousse $\cup$ Gabes & 0.0121 & 0 & 0 & 0 & 0 \\
\hline Monastir $\cup$ Mahdia & 0.0334 & 0 & 0 & 0 & 0 \\
\hline Sidi Bouzid $\cup$ Kasserine & 0.5486 & 0 & 0 & 0 & 0 \\
\hline Sousse $\cup$ Gafsa & 0 & 0 & 0 & 0.0471 & 0 \\
\hline $\begin{array}{l}\text { Monastir } \cup \text { Mahdia } \cup \\
\text { Gabes } \cup \text { Sidi Bouzid } \cup \\
\text { Kasserine }\end{array}$ & 0 & 0 & 0 & 0.7529 & 0 \\
\hline Sousse $\cup$ Mahdia & 0 & 0 & 0 & 0 & 0.1818 \\
\hline Sfax $\cup$ Kairouan & 0 & 0 & 0 & 0 & 0.3637 \\
\hline Gabes $\cup$ Kasserine & 0 & 0 & 0 & 0 & 0.0909 \\
\hline Sidi Bouzid $\cup$ Gafsa & 0 & 0 & 0 & 0 & 0.1818 \\
\hline
\end{tabular}

Subsequently, criteria weight values are calculated using equations 17-20 (Table 8).

Table 8: Criteria weights for the waste treatment center location problem

\begin{tabular}{|c|c|c|c|c|c|}
\hline & $w_{1}$ & $w_{2}$ & $w_{3}$ & $w_{4}$ & $w_{5}$ \\
\hline$w_{k}$ & 0.1730 & 0.1873 & 0.2032 & 0.1812 & 0.2552 \\
\hline
\end{tabular}

We see that the results are reasonable, since the criterion "proximity to urban areas" is far more important than the others. In fact, individuals close to infectious healthcare waste are at a potentially threatening risk, because pathogens, chemical organic and inorganic products, acid gases from stack emissions, fugitive emissions or ash are sources of contamination of air and soil. Similarly, water can be contaminated by pathogens and chemical products; hence there is a risk to the environment and groundwater. For these reasons, the criterion "distance between the waste treatment center and the urban area (the most populated zone in the city)" should be assigned high importance. The least important criterion is the cost, because material advantages should be neglected compared to citizens' health and environment interest. In other words, human and environmental capital is far more important than financial capital. All other criteria weights have intermediate values. 
The obtained weight values are used in modified Murphy's rule in order to combine all belief mass distributions. Thereafter, we transform the obtained overall belief mass into pignistic probabilities BetP using pignistic transformation. The results are as follows:

$\operatorname{Bet} P($ Sousse $)=0.0084 ; \operatorname{Bet} P($ Monastir $)=0.0519 ; \operatorname{Bet} P($ Mahdia $)=0.0793$;

$\operatorname{Bet} P(\operatorname{Sfax})=0.1843 ; \operatorname{Bet} P($ Gabes $)=0.0126 ; \operatorname{Bet} P($ Kairouan $)=0.2392 ;$

$\operatorname{Bet} P($ Sidi Bouzid $)=0.2729 ; \operatorname{Bet} P($ Kasserine $)=0.1458 ; \operatorname{Bet} P($ Gafsa $)=0.0057$.

The different sites are ranked in the decreasing order of their pignistic probabilities. The ranking obtained is:

Sidi Bouzid $>$ Kairouan $>$ Sfax $>$ Kasserine $>$ Mahdia $>$ Monastir $>$ Gabes $>$ Sousse $>$ Gafsa.

This ranking is presented to the Ministry of Environment to help the persons in charge to choose the best site (or more sites, according to their needs and their resources) for the creation of a new waste treatment center of healthcare activities with infectious risk in the center of Tunisia.

\section{Conclusion and further research}

In multi-criteria decision problems, information provided by the decision maker may be incomplete for various reasons. In the literature, there are only few papers solving multi-criteria decision making problems with incomplete information. Most of them discuss the AHP method. The PROMETHEE II-BELIEF approach proposed in this paper is a novel, flexible and systematic method for solving multi-criteria decision problems with incomplete information. It incorporates belief function theory into the PROMETHEE II method in order to take into account the incompleteness of information in the decision matrix as well as in criteria weight values.

Different from most of current methods, the PROMETHEE II-BELIEF approach offers the possibility of solving the problem directly on the basis of its incomplete decision matrix. In addition, it has advantages over the standard PROMETHEE II, on the objective determination way of criteria, weights instead of using values provided directly in a subjective manner. Besides, through the determination of focal elements, this approach allows to reduce considerably the number of pairwise comparisons of alternatives with respect to each criterion.

Incomplete information related to preference function thresholds is an interesting topic and can be further investigated. Another possible line of research is extending this method to the context of group decision making. Further extensions include developing approaches for an incomplete decision matrix with fuzzy and uncertain values. A promising research area is the 
development of a decision support system to automate the problem solving because calculations increase multiplicatively as the number of alternatives and criteria increases.

\section{References}

Abdennadher H., Boujelben M.A., Ben Amor S. (2013), An Extension of PROMETHEE with Evidential Evaluations, Proceedings of the IEEE International Conference on World Congress on Computer and Information Technology (WCCIT'13), DOI: 10.1109/WCCIT.2013. 6618751, Sousse, Tunisia, 1-6.

Ahn B.S. (2015), Extreme Point-based Multi-attribute Decision Analysis with Incomplete Information, European Journal of Operational Research, 240(3), 748-755.

Altieri M.G., Dell'Orco M., Marinelli M., Sinesi S. (2017), Evidence (Dempster - Shafer) Theory-Based Evaluation of Different Transport Modes under Uncertainty: Theoretical Basis and First Findings, Transportation Research Procedia, 27, 508-515.

Behzadian M., Kazemzadeh R.B., Albadvi A., Aghdasi M. (2010), PROMETHEE: A Comprehensive Literature Review on Methodologies and Applications, European Journal of Operational Research, 200, 198-215.

Ben Amor S., Mareschal B. (2012), Integrating Imperfection of Information into the PROMETHEE Multi-criteria Decision Aid Method: A General Framework, Foundations of Computing and Decision Sciences, 37(1), 9-23.

Beynon M.J., Curry B., Morgan P. (2000), The Dempster-Shafer Theory of Evidence: An Alternative Approach to Multi-criteria Decision Modeling, OMEGA, 28(1), 37-50.

Brans J.P., Vincke Ph. (1985), A Preference Ranking Organization Method: The PROMETHEE Method, Management Science, 31, 647-656.

Brans J.P., Vincke Ph., Mareschal B. (1986), How to Select and How to Rank Projects: The PROMETHEE Method, European Journal of Operational Research, 24, 228-238.

Chen L., Zhou X., Xiao F., Deng Y., Mahadevan S. (2017), Evidential Analytic Hierarchy Process Dependance Assessment Methodology in Human Reliability Analysis, Nuclear Engineering and Technology, 49(1), 123-133.

Dempster A.P. (1967), Upper and Lower Probabilities Induced by Multi-valued Mapping, Annals of Mathematical Statistics, 38, 325-339.

Dezert J., Tacnet J.M., Batton-Hubert M., Smarandache F. (2010), Multi-criteria Decision Making Based on DSmT-AHP, Proceedings of Belief 2010 International Workshop on the Theory of Belief Functions, Brest, France.

Dezert J., Han D., Tacnet J.M. (2017), Multi-Criteria Decision-Making with Imprecise Scores and BF-TOPSIS, Proceedings of the 20th IEEE International Conference on Information Fusion (Fusion), Xi'an, China.

Ennaceur A., Elouedi Z., Lefèvre E. (2012), Reasoning under Uncertainty in the AHP Method Using the Belief Function Theory, Advances in Computational Intelligence, 300, 373-382.

Ennaceur A., Elouedi Z., Lefèvre E. (2014), An Extension of the Analytic Hierarchy Process Method under the Belief Function Framework, Information Processing and Management of Uncertainty in Knowledge-Based Systems, 444, 233-242.

Ennaceur A., Elouedi Z., Lefèvre E. (2016), Belief AHP Method - AHP Method with the Belief Function Framework, International Journal of Information Technology \& Decision Making, 15(3), 553-573.

Fan Y., Deer P. (2005), A New Approach on $\rho$ to Decision Making Using Belief Functions under Incomplete Information, Proceedings of the $18^{\text {th }}$ Australian Joint Conference on Advances in Artificial Intelligence (AI'05), Springer Verlag, Berlin, 445-456. 
Fortes I., Mora L'opez L., Morales R., Triguero F. (2006), Inductive Learning Models with Missing Values, Mathematical and Computer Modeling, 44(9-10), 790-806.

Haseli G., Sheikh R., Shib, S.S. (2020), Base-criterion on Multi-criteria Decision-making Method and Its Applications, International Journal of Management Science and Engineering Management, 15(2), 79-88.

Hong T.P., Tseng L.H., Wang S.L. (2002), Learning Rules from Incomplete Training Examples by Rough Sets, Expert Systems with Applications, 22(4), 285-293.

Hsu S.C., Wang T.C. (2011), Solving Multi-criteria Decision Making with Incomplete Linguistic Preference Relations, Expert Systems with Applications, 38(9), 10882-10888.

Hua Z., Gong B., Xu X. (2008), A DS-AHP Approach for Multi-attribute Decision Making Problem with Incomplete Information, Expert Systems with Applications, 34, 2221-2227.

Huang S., Su X., Hu Y., Mahadevan S., Deng Y. (2014), A New Decision-making Method by Incomplete Preferences Based on Evidence Distance, Knowledge-Based Systems, 56, 264-272.

Hyde K., Mayer H.R., Colby C. (2003), Incorporating Uncertainty in the PROMETHEE MCDA Method, Journal of Multi-Criteria Decision Analysis, 12(4-5), 245-259.

Ju Y. (2014), A New Method for Multiple Criteria Group Decision Making with Incomplete Weight Information under Linguistic Environment, Applied Mathematical Modelling, 38(21-22), 5256-5268.

Kahneman D., Slovic P., Tversky A. (1982), Judgment under Uncertainty: Heuristics and Biases, Cambridge University Press, Cambridge.

Kaufman C.J. (1988), The Application of Logical Imputation to Household Measurement, Journal of the Market Research Society, 30, 453-466.

Kim S.H., Ahn B.S. (1997), Group Decision Making Procedure Considering Preference Strength under Incomplete Information, Computer and Operations Research, 24(12), 1101-1112.

Kim S.H., Ahn B.S. (1999), Interactive Group Decision Making Procedure under Incomplete Information, European Journal of Operational Research, 116, 498-507.

Kim S.H., Choi S.H., Kim J.K. (1999), An Interactive Procedure for Multiple Attribute Group Decision Making with Incomplete Information: Range-based Approach, European Journal of Operational Research, 118, 139-152.

Liu W. (2006), Analyzing the Degree of Conflict among Belief Functions, Artificial Intelligence, 170, 909-924.

Moalla Frikha H., Chabchoub H., Martel J.M. (2011), An Interactive Disaggregation Approach Determining the Indifference and the Preference Thresholds of PROMETHEE II, International Journal of Multi-Criteria Decision Making, 1(4), 365-393.

Morad K., Svrcek W.Y., McKay I. (2000), Probability Density Estimation Using Incomplete Data, ISA Transactions, 39(4), 379-399.

Murphy C.K. (2000), Combining Belief Functions when Evidence Conflicts, Decision Support Systems, 29(4), 1-9.

Park K.S., Kim S.H., Yoon Y.C. (1996), Establishing Strict Dominance between Alternatives with Special Type of Incomplete Information, European Journal of Operational Research, 96, 398-406.

Pelissari R., Oliveira M.C., Ben Amor S., Abackerli A.J. (2019), A New FlowSort-based Method to Deal with Information Imperfections in Sorting Decision-making Problems, European Journal of Operational Research, 276, 235-246.

Quinlan J.R. (1993), C4.5: Programs for Machine Learning, Morgan Kaufmanum, San Mateo, CA.

Quinten J.R., Raaijmakers W. (1999), Effectiveness of Different Missing Data Treatments in Surveys with Likert-type Data: Introducing the Relative Mean Substitution Approach, Educational and Psychological Measurement, 59(5), 725-748. 
Ramanathan R., Ganesh L.S. (1994), Group Preference Aggregation Methods Employed in AHP: An Evaluation and an Intrinsic Process for Deriving Members' Weightages, European Journal of Operational Research, 79, 249-265.

Raymond M.R. (1986), Missing Data in Evaluation Research, Evaluation and the Health Profession, 9, 395-420.

Ren J., Lutzen M. (2017), Selection of Sustainable Alternative Energy Source for Shipping: Multicriteria Decision Making under Incomplete Information, Renewable and Sustainable Energy Reviews, 74(C), 1003-1019.

Sen D.K., Datta S., Patel S.K., Mahapatra S.S. (2015), Multi-criteria Decision Making towards Selection of Industrial Robot, Benchmarking: An International Journal, 22(3), 465-487.

Shafer G. (1976), A Mathematical Theory of Evidence, Princeton University Press, New York.

Smets Ph. (2002), Decision Making in a Context Where Uncertainty Is Represented by Belief Functions [in:] R.P. Srivastava, T.J. Mock (eds.), Belief Functions in Business Decisions, Physica-Verlag, Heidelberg, Germany, 17-61.

Tacnet J.M. (2009), Prise en compte de l'incertitude dans l'expertise des risques naturels en montagne par analyse multicritères et fusion d'information, $\mathrm{PhD}$ thesis in Environmental Engineering Sciences, Ecole Nationale Supérieure des Mines, Saint-Etienne, France.

Tacnet J.M., Batton-Hubert M., Dezert J. (2009), Information Fusion for Natural Hazards in Mountains [in:] J. Dezert, F. Smarandache (eds.), Advances and Applications of DSmT for Information Fusion. Collected work. Vol. 3, American Research Press, Rehoboth, USA, 565-659.

Tacnet J.M., Batton-Hubert M., Dezert J. (2010), A Two-step Fusion Process for Multi-criteria Decision Applied to Natural Hazards in Mountains, Proceedings of Belief 2010 Int. Workshop, Brest, France.

Tacnet J.M., Dezert J. (2011), Cautious OWA and Evidential Reasoning for Decision under Uncertainty, The $14^{\text {th }}$ International Conference on Information Fusion Proceedings, Chicago, Illinois, USA, 2074-2081.

Tscheikner-Gratl F., Egger P., Rauch W., Kleidorfer M. (2017), Comparison of Multi-Criteria Decision Support Methods for Integrated Rehabilitation Prioritization, Water, 9(2), 68-79.

Wang J. (2006), Multi-criteria Decision-making Approach with Incomplete Certain Information Based on Ternary AHP, Journal of Systems Engineering and Electronics, 17(1), 109-114.

Weber M. (1987), Decision Making with Incomplete Information, European Journal of Operational Research, 28, 44-57.

Zadeh L. (1979), On the Validity of Dempster's Rule of Combination of Evidence, ERL Memorandum M79/24, University of California, Berkeley, CA. 


\section{Appendices}

\section{Appendix A: Complete decision matrix with some equal evaluations of} alternatives

Table A.1: Focal elements decision matrix $G^{\prime}$ of case 1

\begin{tabular}{|c|c|c|c|c|c|}
\hline & $C_{1}$ & $C_{2}$ & $C_{3}$ & $C_{4}$ & $C_{5}$ \\
\hline$A$ & 20 & 15 & 4 & $*$ & $*$ \\
\hline$B$ & 52 & 20 & 3 & 4 & 4 \\
\hline$C$ & 14 & 35 & 2 & 1 & 2 \\
\hline$D$ & 5 & 63 & $*$ & $*$ & 1 \\
\hline$E$ & 8 & 10 & $*$ & $*$ & 3 \\
\hline$A \cup B$ & $*$ & $*$ & $*$ & 3 & $*$ \\
\hline$D \cup E$ & $*$ & $*$ & $*$ & $*$ & $*$ \\
\hline
\end{tabular}

Table A.2: Belief mass distribution of case 1

\begin{tabular}{|c|c|c|c|c|c|}
\hline & $m_{1}$ & $m_{2}$ & $m_{3}$ & $m_{4}$ & $m_{5}$ \\
\hline$A$ & 0.1767 & 0.2590 & 0.4053 & 0 & 0 \\
\hline$B$ & 0.0942 & 0.2375 & 0.2866 & 0.3717 & 0 \\
\hline$C$ & 0.2111 & 0.1295 & 0.1805 & 0.1858 & 0.3717 \\
\hline$D$ & 0.2684 & 0.0916 & 0 & 0.1475 & 0.1858 \\
\hline$E$ & 0.2496 & 0.2824 & 0 & 0 & 0.1475 \\
\hline$A \cup B$ & 0 & 0 & 0 & 0 & 0.2950 \\
\hline$A \cup E$ & 0 & 0 & 0 & 0.2950 & 0 \\
\hline$D \cup E$ & 0 & 0 & 0.1276 & 0 & 0 \\
\hline
\end{tabular}

Table A.3: Criteria weights of case 1

\begin{tabular}{|c|c|c|c|c|c|}
\hline & $w_{1}$ & $w_{2}$ & $w_{3}$ & $w_{4}$ & $w_{5}$ \\
\hline$w_{k}$ & 0.1987 & 0.2045 & 0.1880 & 0.2102 & 0.1986 \\
\hline
\end{tabular}

\section{Appendix B: Dominant alternative}

Table B.1: Focal elements decision matrix $G^{\prime}$ of case 2

\begin{tabular}{|c|c|c|c|c|c|}
\hline & $C_{1}$ & $C_{2}$ & $C_{3}$ & $C_{4}$ & $C_{5}$ \\
\hline$A$ & 20 & 15 & 3 & $*$ & $*$ \\
\hline $\boldsymbol{B}$ & 4 & 8 & 4 & 4 & $*$ \\
\hline$C$ & 14 & 35 & 2 & 2 & 4 \\
\hline$D$ & 5 & 63 & $*$ & $*$ & 1 \\
\hline$E$ & 8 & 10 & $*$ & $*$ & 5 \\
\hline$A \cup B$ & $*$ & $*$ & $*$ & 3 & $*$ \\
\hline$D \cup E$ & $*$ & $*$ & $*$ & $*$ & $*$ \\
\hline
\end{tabular}


Table B.2: Belief mass distribution of case 2

\begin{tabular}{|c|c|c|c|c|c|}
\hline & $m_{1}$ & $m_{2}$ & $m_{3}$ & $m_{4}$ & $m_{5}$ \\
\hline$A$ & 0.1366 & 0.2375 & 0.2866 & 0 & 0 \\
\hline$B$ & 0.2408 & 0.2824 & 0.4053 & 0.3717 & 0 \\
\hline$C$ & 0.1725 & 0.1295 & 0.1805 & 0.1858 & 0.3579 \\
\hline$D$ & 0.2346 & 0.0916 & 0 & 0.1475 & 0.1421 \\
\hline$E$ & 0.2155 & 0.2590 & 0 & 0 & 0.1421 \\
\hline$A \cup B$ & 0 & 0 & 0 & 0 & 0.3579 \\
\hline$A \cup E$ & 0 & 0 & 0 & 0.2950 & 0 \\
\hline$D \cup E$ & 0 & 0 & 0.1276 & 0 & 0 \\
\hline
\end{tabular}

Table B.3: Criteria weights of case 2

\begin{tabular}{|c|c|c|c|c|c|}
\hline & $w_{1}$ & $w_{2}$ & $w_{3}$ & $w_{4}$ & $w_{5}$ \\
\hline$w_{k}$ & 0.2020 & 0.2020 & 0.1916 & 0.2123 & 0.1921 \\
\hline
\end{tabular}

\section{Appendix C: Dominated alternative}

Table C.1: Focal elements decision matrix $G$ ' of case 3

\begin{tabular}{|c|c|c|c|c|c|}
\hline & $C_{1}$ & $C_{2}$ & $C_{3}$ & $C_{4}$ & $C_{5}$ \\
\hline$A$ & 20 & 15 & 4 & $*$ & $*$ \\
\hline$B$ & 52 & 20 & 3 & 4 & $*$ \\
\hline$C$ & 14 & 35 & 2 & 2 & 4 \\
\hline$D$ & 55 & 63 & $*$ & 1 & 1 \\
\hline$E$ & 8 & 10 & $*$ & $*$ & 2 \\
\hline$A \cup B$ & $*$ & $*$ & $*$ & $*$ & 3 \\
\hline$A \cup E$ & $*$ & $*$ & $*$ & 3 & $*$ \\
\hline$D \cup E$ & $*$ & $*$ & 1 & $*$ & $*$ \\
\hline
\end{tabular}

Table C.2: Belief mass distribution of case 3

\begin{tabular}{|c|c|c|c|c|c|}
\hline & $m_{1}$ & $m_{2}$ & $m_{3}$ & $m_{4}$ & $m_{5}$ \\
\hline$A$ & 0.2304 & 0.2590 & 0.4053 & 0 & 0 \\
\hline$B$ & 0.1101 & 0.2375 & 0.2866 & 0.3717 & 0 \\
\hline$C$ & 0.2594 & 0.1295 & 0.1805 & 0.1858 & 0.3717 \\
\hline$D$ & 0.1083 & 0.0916 & 0 & 0.1475 & 0.1475 \\
\hline$E$ & 0.2918 & 0.2824 & 0 & 0 & 0.1858 \\
\hline$A \cup B$ & 0 & 0 & 0 & 0 & 0.2950 \\
\hline$A \cup E$ & 0 & 0 & 0 & 0.2950 & 0 \\
\hline$D \cup E$ & 0 & 0 & 0.1276 & 0 & 0 \\
\hline
\end{tabular}

Table C.3: Criteria weights of case 3

\begin{tabular}{|c|c|c|c|c|c|}
\hline & $w_{1}$ & $w_{2}$ & $w_{3}$ & $w_{4}$ & $w_{5}$ \\
\hline$w_{k}$ & 0.2047 & 0.2082 & 0.1873 & 0.2026 & 0.1972 \\
\hline
\end{tabular}




\section{Appendix D: Incomplete information}

Table D.1: Focal elements decision matrix $G^{\prime}$ of case 4

\begin{tabular}{|c|c|c|c|c|c|}
\hline & $C_{1}$ & $C_{2}$ & $C_{3}$ & $C_{4}$ & $C_{5}$ \\
\hline$A$ & 20 & 15 & $*$ & 3 & 4 \\
\hline$B$ & 52 & $*$ & 3 & $*$ & 3 \\
\hline$C$ & 14 & 35 & 2 & 2 & $*$ \\
\hline$D$ & 5 & 63 & 4 & 1 & 2 \\
\hline$E$ & $*$ & 10 & 1 & 4 & 1 \\
\hline
\end{tabular}

Table D.2: Belief mass distribution of case 4

\begin{tabular}{|c|c|c|c|c|c|}
\hline & $m_{1}$ & $m_{2}$ & $m_{3}$ & $m_{4}$ & $m_{5}$ \\
\hline$A$ & 0.2449 & 0.3317 & 0 & 0.2865 & 0.3407 \\
\hline$B$ & 0.1420 & 0 & 0.2818 & 0 & 0.2865 \\
\hline$C$ & 0.2754 & 0.1972 & 0.1992 & 0.2026 & 0 \\
\hline$D$ & 0.3377 & 0.1394 & 0.3654 & 0.1703 & 0.2026 \\
\hline$E$ & 0 & 0.3317 & 0.1536 & 0.3407 & 0.1703 \\
\hline
\end{tabular}

Table D.3: Criteria weights of case 4

\begin{tabular}{|c|c|c|c|c|c|}
\hline & $w_{1}$ & $w_{2}$ & $w_{3}$ & $w_{4}$ & $w_{5}$ \\
\hline$w_{k}$ & 0.1945 & 0.2103 & 0.1837 & 0.2147 & 0.1968 \\
\hline
\end{tabular}

